\title{
Strong asymmetrical inter-specific relationships in food web simulations
}

\author{
Ferenc Jordán ${ }^{1,2, *}$, Marco Scotti ${ }^{1,3}$, Ágnes Mike ${ }^{4}$, Marco Ortiz $^{5}$ \\ ${ }^{1}$ The Microsoft Research - University of Trento Centre for Computational and Systems Biology, Piazza Manifattura 1, \\ 38068 Rovereto, TN, Italy \\ ${ }^{2}$ MTA Centre for Ecological Research, Balaton Limnological Institute, Tihany, Hungary \\ ${ }^{3}$ GEOMAR Helmholtz-Zentrum für Ozeanforschung, Duesternbrooker Weg 20, 24105 Kiel, Germany \\ ${ }^{4}$ Center for Physiology and Pharmacology, Department of Neurophysiology and Pharmacology, \\ Medical University of Vienna, Vienna, Austria \\ ${ }^{5}$ Instituto Antofagasta, Instituto de Ciencias Naturales AvH, Facultad de Ciencias del Mar \& Recursos Biológicos, \\ Universidad de Antofagasta, Antofagasta, Chile
}

\begin{abstract}
In complex ecosystem models, relationships between species include a large number of direct interactions and indirect effects. In order to unveil some simple and better understandable relationships, it is useful to study the asymmetry of inter-specific effects. We present a simple approach for this based on stochastic food web simulations from previous studies. We refer to the Prince William Sound (Gulf of Alaska) marine ecosystem model for illustration. Real data were used to parameterize a dynamical food web model. Through simulations and sensitivity analysis, we determined the strength of the effects between all species. We calculated the asymmetry between the mutual effects species have on each other, and selected the top $5 \%$ most asymmetrical interactions. The set of these highly asymmetrical relationships is illustrated by a separate graph in which we calculated the positional importance of the species and correlated this to other independent properties such as population size and trophic position. Results suggest that halibut is the key species dominating this system of asymmetrical interactions, but sablefish and adult arrowtooth flounder also seem to be of high importance. Nearshore demersals display the highest number of connections in the graph of asymmetrical links, suggesting that this trophic group regulates the dynamics of many species in the food web. This approach identifies key interactions and most asymmetrical relationships, potentially increasing the efficiency of management efforts and aiding conservation efforts.
\end{abstract}

KEY WORDS: Food web - Stochastic simulation · Asymmetrical effects · Prince William Sound · Eco-dynamics

\section{INTRODUCTION}

Population biology and the study of pairwise interspecific interactions provide detailed information about community dynamics. At a larger scale, ecosystem studies describe the system-level, macroscopic properties of nature (Lindeman 1942). The objectives of macroscopic descriptions are simplification (e.g. network unfolding, Whipple \& Patten 1993; dominator tree, Allesina \& Bodini 2004), cross-system comparability (Baird \& Ulanowicz 1993), and generalization (Ulanowicz \& Kemp 1979, Hirata \& Ulanowicz 1985). It is both important and challenging to try to connect the 2 levels, i.e. to understand the role individual species and single interactions play in the whole ecosystem (Jones \& Lawton 1995). Studies on keystone species (Power et al. 1996, Libralato et al. 2006, Jordán 2009, Ortiz et al. 2013) and ecological redundancy (Bond 1994) provide some useful background for how to approach this problem. Related 
studies have quantified the similarity between the roles species play in ecological systems, based on topological equivalence measures (regular equivalence, Luczkovich et al. 2003), Jaccard-similarity of feeding (trophospecies, Yodzis \& Winemiller 1999), or the overlap of the neighborhood (trophic overlap, Jordán et al. 2009, Lai et al. 2012). For simplicity, hereafter we will make use of the term 'species' as an equivalent of 'trophic group' and 'component.'

Large ecosystem models found in modern online databases are highly complex, especially when indirect effects are accounted for. Due to this complexity, in many trophic network models it is difficult to examine whether species $x$ has a larger effect on species $y$ or vice versa, and the study of their flow structure contributes to unveiling unexpected beneficial effects of predators upon their prey (Ulanowicz \& Puccia 1990, Bondavalli \& Ulanowicz 1999). Several methods of quantitative modeling have been used for determining key species and interactions, ranging from loop analysis (Briand \& McCauley 1978, Bodini 1991, Ortiz \& Wolff 2002, Ortiz \& Levins 2011) to indices based on flow analysis (Hirata \& Ulanowicz 1985, Vasas et al. 2007, Borrett 2013). These near-equilibrium approaches provide qualitative or quantitative results and predictions.

Structural models can be complemented by dynamical analysis, either of analytical nature or using simulations with realistic parameters. In certain cases, mass equations can be used to realistically describe eco-dynamics (e.g. phytoplankton blooms), but these approaches make unrealistic assumptions when applied to the dynamics of small populations of largebodied animals. The latter case calls for the use of individual-based techniques (Melián et al. 2011), which incorporate information on demography, building better links between ecological and evolutionary processes and offering a better view on rare species. A complementary way to investigate the mechanistic details of trophic dynamics is based on individualbased simulations (Kazanci et al. 2009, Livi et al. 2011). These approaches need to be integrated in order to better understand how local effects (particular species and interactions) scale up and shape global eco-dynamics.

In this paper, we present a modeling and simulation framework that helps to identify and quantify the most asymmetrical relationships between the species of a marine ecosystem. We show and discuss the most asymmetrical inter-specific relationships (direct interactions and indirect effects) in the Prince William Sound ecosystem and, based on these, we present a graph of asymmetrical relationships of the ecosys- tem, inspired by the backbone of trophic transfers suggested by Lindeman (1942) (the Lindeman spine; see Baird \& Ulanowicz 1993). Most importantly, many of these relationships exist between species directly unlinked in the food web. Instead of focusing on key species, we identify the key interactions in the ecosystem. This approach has been seriously underemphasized in the literature. Finally, we discuss the indicator value of this method for conservation and management.

\section{METHODS}

\section{Study system}

The Prince William Sound (Gulf of Alaska) ecosystem was described in great detail (Okey \& Pauly 1999) and modeled by the EcoPath with EcoSim (Christensen \& Walters 2004) toolkit in order to determine key species by sensitivity analysis (Okey \& Wright 2004). The trophic network model contains 48 living groups, and the architecture of preypredator interactions defines the food web topology (i.e. the structure of the trophic network). The weighted version of the food web is also available; interaction strengths are quantified in terms of biomass flows (US tons of wet weight $\mathrm{km}^{-2} \mathrm{yr}^{-1}$; see Okey 2004).

\section{Food web}

Between the $N=48$ species there are $L=355$ realized trophic interactions (prey-predator carbon transfers). This corresponds to $2 \mathrm{~L}=710$ realized direct pairwise effects (both bottom-up and top-down between the prey-predator pairs of species). If indirect effects are also considered, almost everything can affect everything else, so we can list $N(N-1)=$ 2256 directed pairwise effects and $N(N-1) / 2=1128$ undirected pairwise relationships in the community (i.e. only self-loop effects are excluded). Our aim is to identify the most important directed pairwise relationships that dominate the dynamics of the ecosystem. The key of our methodology is (1) to structurally quantify the strength of pairwise interactions and the asymmetry of pairwise effects (using the binary structure of food web interactions, but also referring to the weighted food webs, with interaction strength quantified through carbon transfers), (2) to simulate network dynamics and also dynamically quantify the strength and asymmetry of these inter-specific effects, 
(3) to single out the most asymmetrical simulated effects, and (4) to study the network position of species in the graph of the most asymmetrical relationships and characterize the properties of these most asymmetrical links.

\section{Network analysis: structure}

Several centrality indices have been used to quantify the positional (i.e. structural) importance of species in food webs. From the simplest ones (e.g. node degree, Wasserman \& Faust 1994) to more complicated ones (i.e. communicability, Estrada 2007), there is a range of possibilities to choose the appropriate tool for addressing a particular problem. Since we also focus on the importance of interactions starting from the food web topology, we decided to use the topological importance index (TI): this allows quantification of the structural importance (centrality) of network nodes and of the relative strength and asymmetry of the interactions (see Jordán et al. 2003). For this index, we define $a_{n, i j}$ as the effect of $j$ on $i$ when $i$ can be reached from $j$ in $n$ steps. The simplest method of calculating $a_{n, i j}$ is when $n=1$ (i.e. the effect of $j$ on $i$ in 1 step): $a_{1, i j}=1 / D_{i}$, where $D_{i}$ is the degree of node $i$ (i.e. the number of its direct neighbors including both prey or predatory species). We assume that indirect chain effects are multiplicative and additive. For instance, if we were to determine the effect of $j$ on $i$ in 2 steps in a hypothetical network with two 2-step pathways from $j$ to $i$ : one is through $k$ and the other is through $h$. The effect of $j$ on $i$ through $k$ is defined as the product of 2 direct effects (i.e. $a_{1, k j}$ $\left.\times a_{1, i k}\right)$, hence the term 'multiplicative.' Similarly, the effect of $j$ on $i$ through $h$ equals $a_{1, h j, 1} \times a_{1, i h}$. To determine the 2-step effect of $j$ on $i\left(a_{2, i j}\right)$, we simply sum up those 2 individual 2-step effects (i.e. $a_{2, i j}=a_{1, k j} \times$ $\left.a_{1, i k}+a_{1, h j} \times a_{1, i h}\right)$, hence the term 'additive.'

When the effect of step $n$ is considered, we define the effect received by species $i$ from all species in the same network as:

$$
\psi_{n, i}=\sum_{j=1}^{N} a_{n, i j}
$$

which is equal to 1 (i.e. each species is affected by the same unit effect). Furthermore, we define the $n$-step effect originating from species $i$ as:

$$
\sigma_{n, i}=\sum_{j=1}^{N} a_{n, j i}
$$

which may vary among different species (i.e. effects originating from different species can be different).
We define the topological importance of species $i$ when effects up to $n$ steps are considered as:

$$
\mathrm{TI}^{n}{ }_{i}=\frac{\sum_{m=1}^{n} \sigma_{m, i}}{n}=\frac{\sum_{m=1}^{n} \sum_{j=1}^{N} a_{m, j i}}{n}
$$

which is simply the sum of effects originating from species $i$ up to $n$ steps $\left(1+2+3 \ldots\right.$ up to $n_{i} m=$ number of steps) averaged by the maximum number of steps considered (i.e. $n$ ).

Between each ij ordered pair of nodes, the topological constraint on the strength and the $\mathrm{TI}_{\text {asymm(ij) }}^{3}$ asymmetry of the interaction were calculated (their structural connectedness). We used the TI index for up to 3 step long indirect effects $\left(\mathrm{TI}^{3}\right)$ : 3 steps are generally considered to be a meaningful and relevant range for indirect effects in food webs, with typically no major difference found for longer pathways (e.g. Jordán et al. 2003, Brose et al. 2005).

For the weighted version of the network, we used the mixed trophic impact analysis (Ulanowicz \& Puccia 1990). We measured direct and indirect trophic impacts from trophic flow data. The dietary coefficient $g_{i j}$ quantifies the effect of prey $i$ upon predator $j$ (defined as the proportion of $i$ within the menu of $j$ ). The effect of predator $j$ on its prey $i\left(f_{j i}\right)$ is measured by the fraction of net output from prey $i$ (respiratory output is excluded) that is consumed by predator $j$. The net impact of $i$ upon $j$ equals $g_{i j}-f_{j i}$ and is defined as the 1-step (direct) effect of $i$ on $j$. Its values range from -1 to +1 . Taking every pair of the $N$ nodes, we calculated the direct net impacts and computed the $N \times N$ net impact matrix, [Q]. The total (direct and indirect) is summarized in the mixed trophic impact matrix [M] that is calculated by summing up all the integer powers $h$ of [Q]:

$$
[\mathbf{M}]=\sum_{\mathrm{h}=1}^{\infty}[\mathbf{Q}]^{\mathrm{h}}
$$

using the following equation known from the inputoutput theory:

$$
[\mathbf{M}]=\{[\mathbf{I}]-[\mathbf{Q}]\}^{-1}-[\mathbf{I}]
$$

where [I] is the identity matrix. Summing the rows of [M] provides a measure of node importance: we considered the absolute values of effects (called IMA) in order to quantify the interactive power of a group (Vasas \& Jordán 2006). Keystones can be of high importance because of both positive and negative effects on others. Since summing up strong negative and strong positive effects might result in an aggregate value around 0 , we considered absolute values of effects. 


\section{Network simulation: dynamics}

For dynamical simulations, we used the BlenX process algebra language and stochastic simulation platform (Dematté et al. 2008) already adapted for simulating marine food web dynamics (Jordán et al. 2011). To calculate the mutual sensitivity of all species, we built a balanced food web model, simulated it using a master parameter set, and then used that as the baseline for our sensitivity analysis. All reference simulation runs were sampled at time $t$. At time $t$, the mean population size of all species was registered, based on $R$ simulations. To estimate the effect of species $i$ on the mean population size of species $j$, we first defined the reference value of population density for species $j\left(A_{j}\right)$, in the absence of any perturbation (i.e. the initial population size of each species was set according to the value estimated from empirical data, and then simulations were carried out; see Okey 2004):

$$
A_{j}=\frac{\sum_{k=1}^{R} a_{k, j}(t)}{R}
$$

where $R$ simulations are performed, and, for each run $k$, the population size of species $j$ in the undisturbed system $\left(a_{k, j}\right)$ is recorded at time $t$. As a perturbation, the initial number of individuals belonging to a given species was halved, and the mean population size of all other species recorded after time $t$. For each species in the food web, we applied the same perturbation by carrying out $R$ simulations (we note that other disturbance regimes can also be studied, see Scotti et al. 2012). The value of population density for species $j$ after disturbing species $i$ is:

$$
A_{j}(i)=\frac{\sum_{k=1}^{R} a_{k, j}(i)(t)}{R}
$$

and the relative response (rr) of species $j$ to disturbing species $i$ is:

$$
\operatorname{rr}(i)=\frac{\left|A_{j}-A_{j}(i)\right|}{A_{j}}
$$

The relative response is normalized (nrr) over all species $(N)$ :

$$
\operatorname{nrr}_{j}(i)=\frac{\operatorname{rr}_{j}(i)}{\sum_{i=1}^{N} \operatorname{rr}_{j}(i)}
$$

The community importance of species $i$ equals:

$$
I_{H}(M)_{i}=\sum_{j=1}^{N} \operatorname{nrr}_{j}(i)
$$

The normalized relative response metric refers to changes on average population sizes $(M)$; it measures the sensitivity of the system to disturbing component $i$ ( $I_{H}$, where $H$ stands for the Hurlbert response function: Hurlbert 1997). These simulation-based values are dynamical measurements of community-level importance. The Hurlbert response index was already used with the Prince William Sound network model for identifying the most vulnerable and most dominating species (Livi et al. 2011), quantifying indirect interaction modules (Gjata et al. 2012), and characterizing weak interactions (Scotti et al. 2012). The individual effect of species $i$ on species $j$ corresponds to $e_{i j}$, the largest value of each pair of $e_{i j}$ and $e_{j i}$ effects corresponds to maxe $e_{i j}$, and the simulated asymmetry is:

$$
C_{i j}=\left|e_{i j}-e_{j i}\right|
$$

which is the absolute value of the difference between the 2 simulated effects between 2 species.

We identified the most asymmetrical $C_{i j}$ values (i.e. top $5 \%$ ). We did not consider relationships that are strong in both directions: this kind of symmetry makes inter-specific relationships unclear and unpredictable, while asymmetrical relationships may provide more insights about the flow of indirect effects in ecosystems.

It is important to emphasize that any type of effect (weak or strong, symmetrical or asymmetrical) can be important in nature and also during the simulation experiments. For example, the potential importance of weak links has been reported several times (McCann et al. 1998, Scotti et al. 2012). Here, we only aimed to simplify complexity by looking at the clearest relationships (most asymmetrical, typically strong in one direction and weak in the other), as suggested by stochastic simulations. Note that asymmetry has also been used in a different sense in the literature, describing the relative importance of energy channels (Rooney et al. 2006, Rooney \& McCann 2012).

In the graph of the most asymmetrical relationships, we quantify the positional importance of individual nodes by directed topological importance up to 3 steps $\left(\mathrm{TI}^{3}\right)$. In this network, we can define source, sink, and intermediate as well as isolated nodes. We emphasize the difference between our view on dominance and the major alternative approach based on the dominator tree. The dominator tree (Allesina \& Bodini 2004) considers only bottom-up effects (i.e. it relies on a donorbased perspective), it is a static analysis, and it is based only on direct interactions. The dominance measure we suggest is based on simulations and considers indirect effects spreading in any direction (including topdown). Most importantly, the graph of the most asymmetrical relationships is not necessarily a tree. 


\section{Relationships between structure and dynamics}

Finally, we compared the centrality of species $\left(\mathrm{TI}^{3}\right)$ in the graph of the most asymmetrical relationships with other properties: (1) trophic position (TP); (2) the Hurlbert response function related to changes in the population size of each species $\left(I_{H}(M)\right)$; (3) total direct and indirect effects estimated using the weighted food web (IMA); (4) population size, on a natural logarithmic scale $(\ln (N))$. The effective TP index characterizes the trophic height of individual species (Okey 2004). TP stands for the weighted average distance of species from primary producers (Scotti et al. 2006) and is computed as the sum of the fractions of trophic activity that each species performs at different (discrete) trophic levels.

\section{RESULTS}

By selecting only the top 5\% most asymmetrical relationships, 56 out of 1128 potential effects were included in the asymmetry graph. Fig. 1 shows the rank of asymmetry values and marks interactions

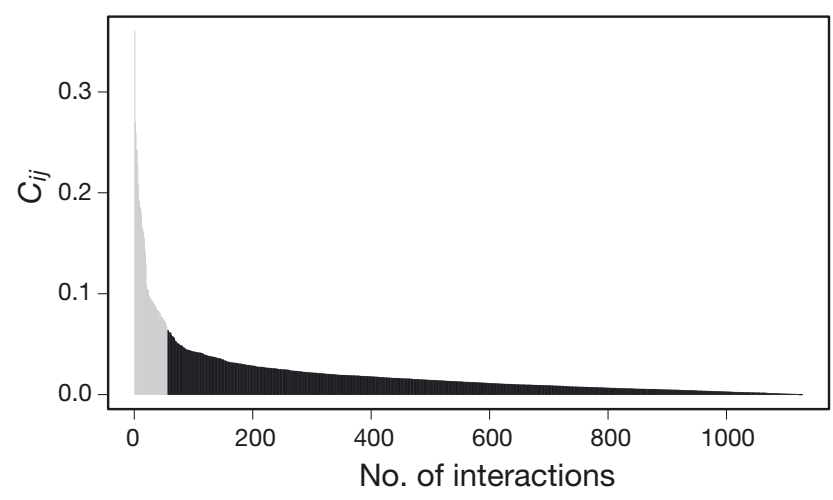

Fig. 1. Rank of the asymmetry values for all ij interactions, according to stochastic food web simulations. The most asymmetrical relationships (i.e. 56 interactions that represent the top $5 \%$ ) are shown in grey and appear in the asymmetry graph (Fig. 2). Less asymmetrical relationships are shown in black; these are not part of this network. The $y$-axis shows the asymmetry values of $i j$ and $j i$ effects quantified by the $C_{i j}$ index. See 'Methods' for definitions of terms

over and below the $5 \%$ threshold with different shades. Fig. 2 shows the graph of the most asymmetrical relationships. The graph is composed of a large component of 42 species, while 6 isolated species are

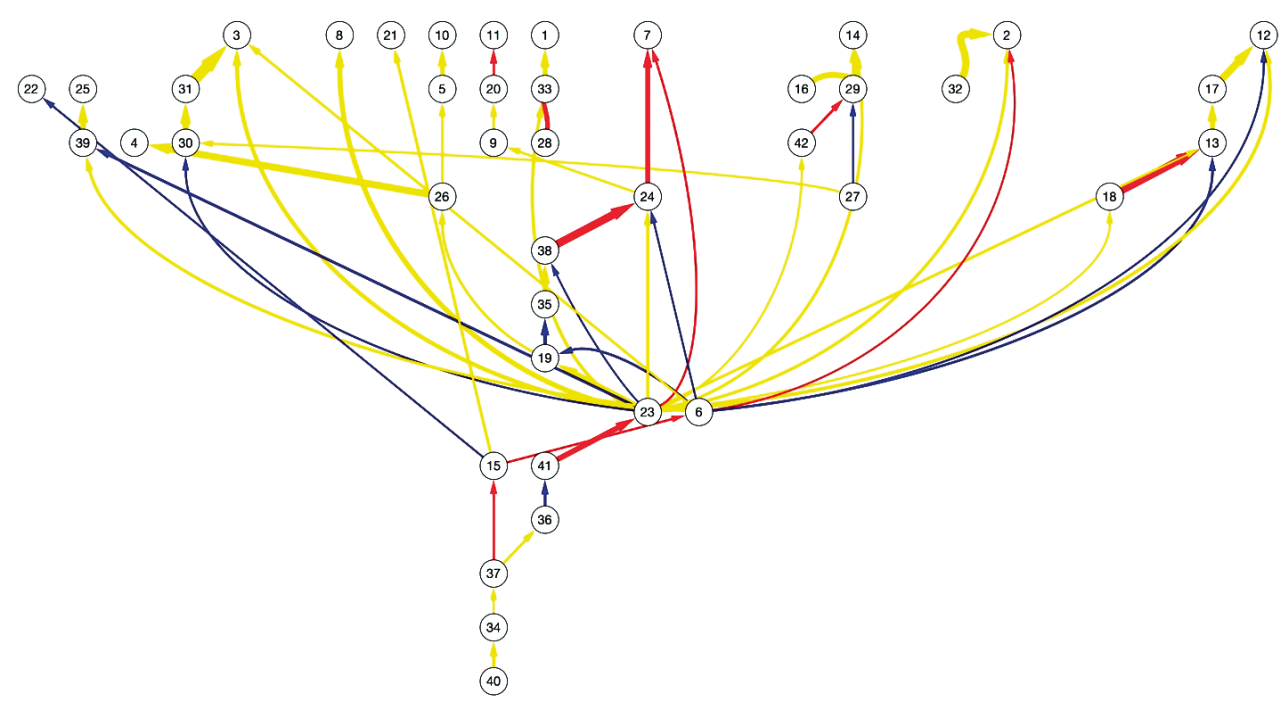

Fig. 2. Asymmetry graph. Interactions of the 56 most asymmetrical relationships (i.e. top $5 \%$; grey area in Fig. 1) for the Prince William Sound ecosystem, estimated by stochastic simulations. This hierarchical layout shows the species at the bottom who are at the root of the clearest relationships. Note that the interactions are directed here, so we only consider the stronger one of the pair of $i j$ and $j i$ effects (see 'Methods' for definitions of terms). Isolated nodes (shallow large epibenthos, deep epibenthos, sea otters, pinnipeds, sleeper sharks, and resident orca) with no strongly asymmetrical interactions are not depicted. Interaction width is proportional to the strength of asymmetry; colors represent different types of effects: blue = bottom-up, red = topdown, yellow $=$ indirect. Node codes (ordered according to trophic position of species, starting from primary producers): $1=$ macroalgae; 2 = offshore phytoplankton; 3 = nearshore phytoplankton; 4 = nearshore herbivorous zooplankton; $5=$ shallow large infauna; $6=$ herbivorous zooplankton; $7=$ shallow small epibenthos; $8=$ deep large infauna; $9=$ meiofauna; $10=$ shallow small infauna; $11=$ omnivorous zooplankton; $12=$ deep small infauna; $13=$ nearshore omnivorous zooplankton; $14=$ jellies; $15=$ juvenile herring; $16=$ sandlance; $17=$ invertebrate-eating birds; $18=$ age 0 pollock; $19=$ adult herring; $20=$ capelin; $21=$ eulachon; 22 =squid; $23=$ nearshore demersals; $24=$ salmon fry $0-12 \mathrm{~cm} ; 25=$ baleen whales; $26=$ rockfish; $27=$ age $1+$ pollock; $28=$ deep demersals; $29=$ seabirds; $30=$ octopods; $31=$ avian predators (eagles and falcons); $32=$ spiny dogfish; $33=$ juvenile arrowtooth; $34=$ sablefish; $35=$ pacific cod; $36=$ adult salmon; $37=$ adult arrowtooth; $38=$ lingcod; $39=$ porpoise $; 40=$ halibut; $41=$ salmon sharks; $42=$ transient orca 
not involved in any strongly asymmetrical relationship (i.e. shallow large epibenthos, deep epibenthos, sea otters, pinnipeds, sleeper sharks, and resident orca). This network is a directed acyclic graph (DAG). However, by applying the same analytical approach to other food webs, we might not necessarily obtain DAGs. The major source of the DAG is halibut, so this fish is proposed to ultimately dominate the asymmetrical relationships in the ecosystem (for its keystone role, see Seitz et al. 2007). Other minor source nodes include spiny dogfish, age 1+ pollock, and deep demersals. These species are not strongly influenced by others. Important sinks include jellies, baleen whales, and squid: these groups have no major asymmetrical effect on others, but some of them are strongly influenced by a series of asymmetrical links.

From a food web perspective, the most asymmetrical links can be quite mixed: the longest chain (8 steps in length) in the asymmetry graph starts with halibut that dominates sablefish. These 2 species are linked through an indirect effect. The following interactions, after sablefish, involve adult arrowtooth (indirect), juvenile herring (top-down), herbivorous zooplankton (top-down), adult herring (bottom-up), rockfish (indirect), shallow large infauna (indirect), and shallow small infauna (indirect).

Fig. 3 presents a classification of the most asymmetrical interactions. Direct bottom-up (blue) and top-down (red) as well as indirect (yellow) effects are marked differently. We tested for a positive association between effect type and asymmetry by applying a 1-tailed Fisher's exact test and found no positive correlation between effect type (bottom-up, $p=0.323$; top-down, $\mathrm{p}=0.133$; indirect, $\mathrm{p}=0.946$ ) and the asymmetry graph.

Fig. 4 shows how $C_{i j}$ is correlated with $\max e_{i s}$ (Fig. 4a), the asymmetry of $\mathrm{TI}^{3}$ effects (Fig. 4b), and mixed trophic impact (Fig. 4c). Strong simulated interactions are generally more asymmetrical as well (Fig. 4a), but strong structural asymmetry does not predict dynamical asymmetry (Fig. 4b), also when the information concerning the strength of biomass flows is considered (Fig. 4c). For a more complete comparison of the relationships between structural and dynamical indices, see Jordán et al. (2008). Based on Fig. 4, large structural asymmetries can be dynamically symmetrical, and structurally symmetrical relationships can result in dynamically highly asymmetrical interactions. If the simulation model is correct, this may show the limitations of topological studies on food webs. It is of interest to determine the trophic height of key species (Scotti \& Jordán 2010). We observed a significant and positive correlation between species importance in the asymmetry graph and TP (Spearman's rho $=0.494 ; \mathrm{p}=0.001$; see Fig. 5a). This illustrates how species feeding higher in the trophic chain may play a possible central role in eco-dynamics. However, species at intermediate trophic positions are involved in strongly asymmetrical relationships as well (Fig. 5a). In our simulations, we observe a certain top-down dominance of community control, even if this trend is not confirmed by statistical analysis (Fisher's exact test, $\mathrm{p}=0.133$ ). From a conservation perspective, it is interesting to note how important rare (meaning 'small population size') species are in eco-dynamics. In this analysis, we did not identify any significant correlation between population size

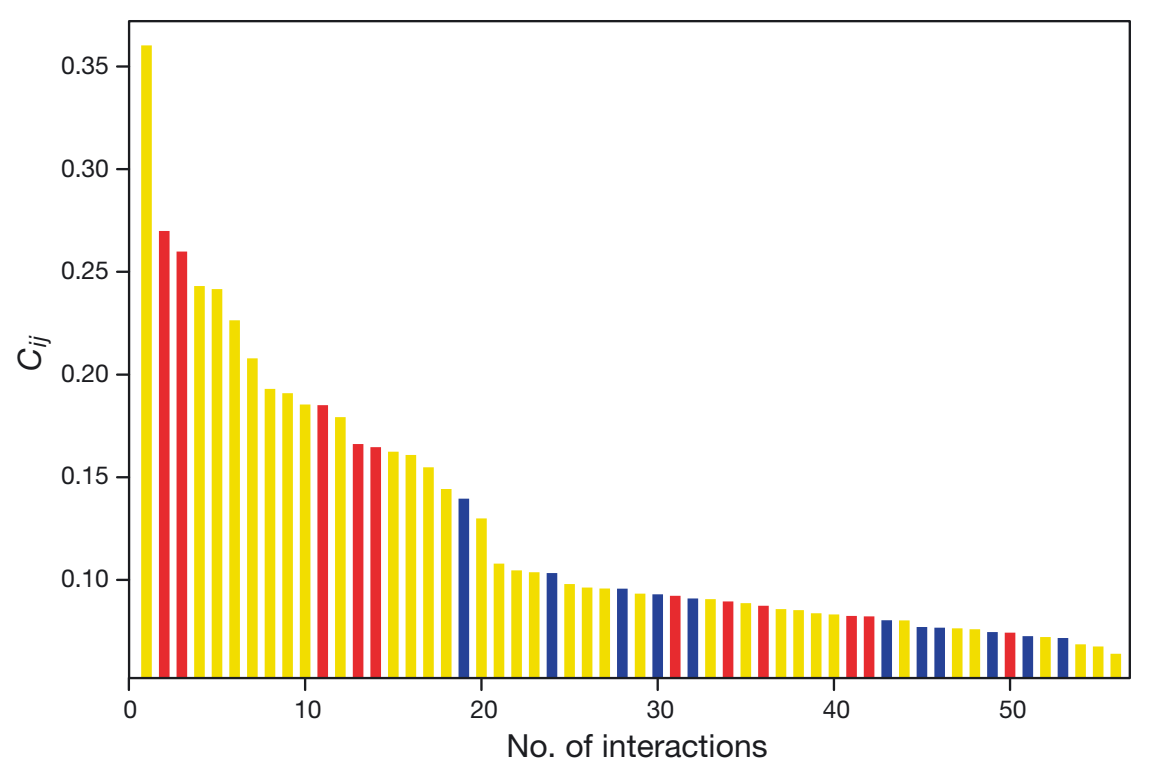

Fig. 3. The 56 most asymmetrical effects (i.e. top $5 \%$ ), ranked and classified according to whether they are indirect (yellow) or direct (bottom-up in blue, top-down in red). Based on simulations, the most asymmetrical relationships are not significantly associated with any of these categories. Indirect effects include all of the $i j$ effects that are not shown directly in the food web (e.g. trophic cascade, exploitative and apparent competition). The $y$-axis shows the $C_{i j}$ asymmetry values of $i j$ and $j i$ effects. This figure represents a zoom of the grey area in Fig. 1. See

'Methods' for definitions of terms 

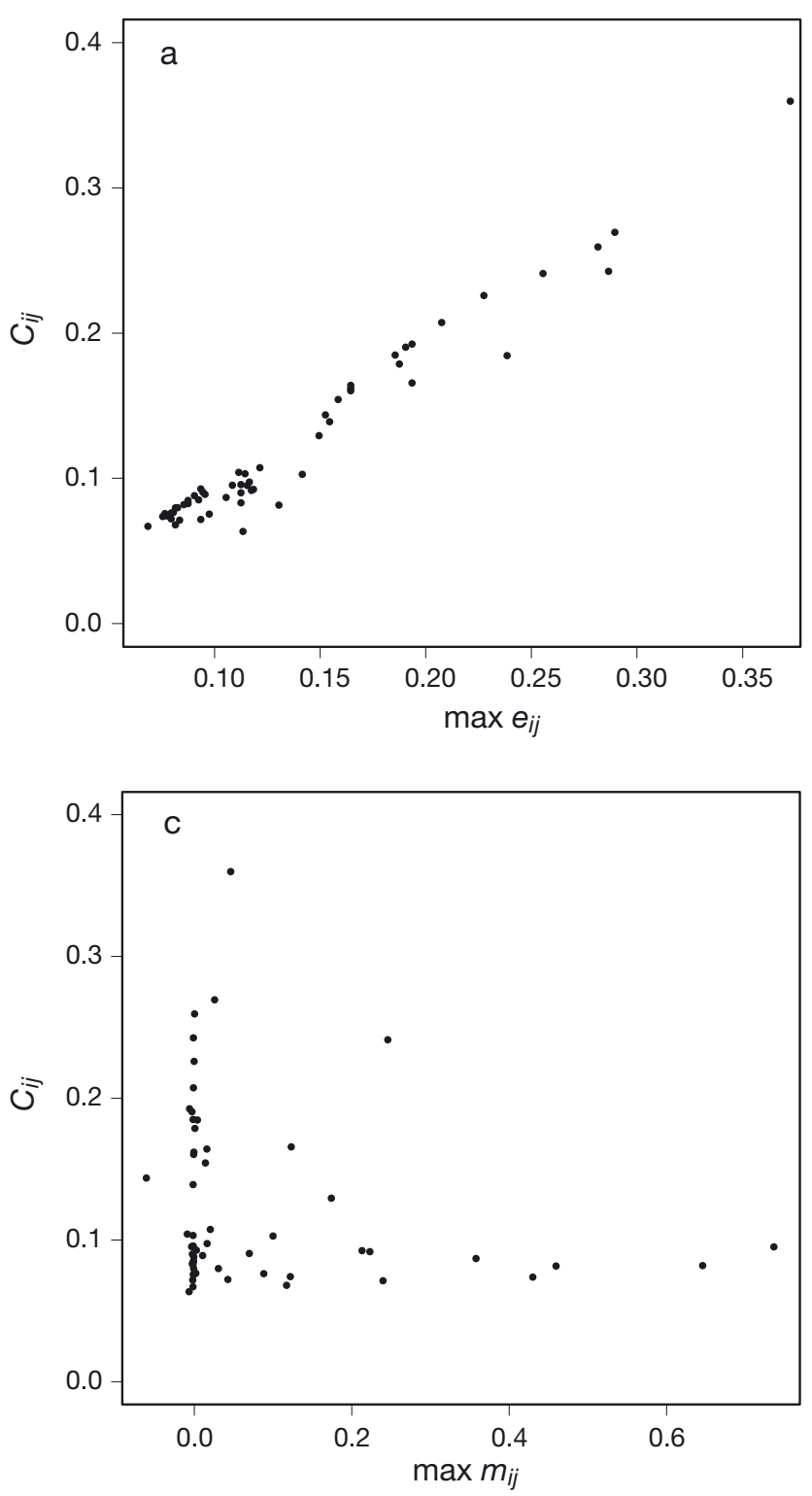

and importance of species in the asymmetry graph (Spearman's rho $=-0.204 ; \mathrm{p}=0.214 ;$ see Fig. $5 \mathrm{~d}$ ).

We can also see that the $\mathrm{TI}^{3}$ of nodes in the asymmetry graph is correlated with their effective TP (Fig. 5a) and the simulated importance $\left(I_{H(M)}\right)$ (Fig. 5b), while no clear and significant patterns link the position of species in the asymmetry graph to the relevance estimated through the mixed trophic impact (IMA; Fig. 5c) and the natural logarithm of population size $(\ln (N)$; Fig. 5d). The importance of species increases with trophic height, but many species at intermediate trophic positions may also be relevant. No clear evidence connects the population size of species to the asymmetry graph. Being rare does not automatically translate into a high asymmetrical simulated impact.

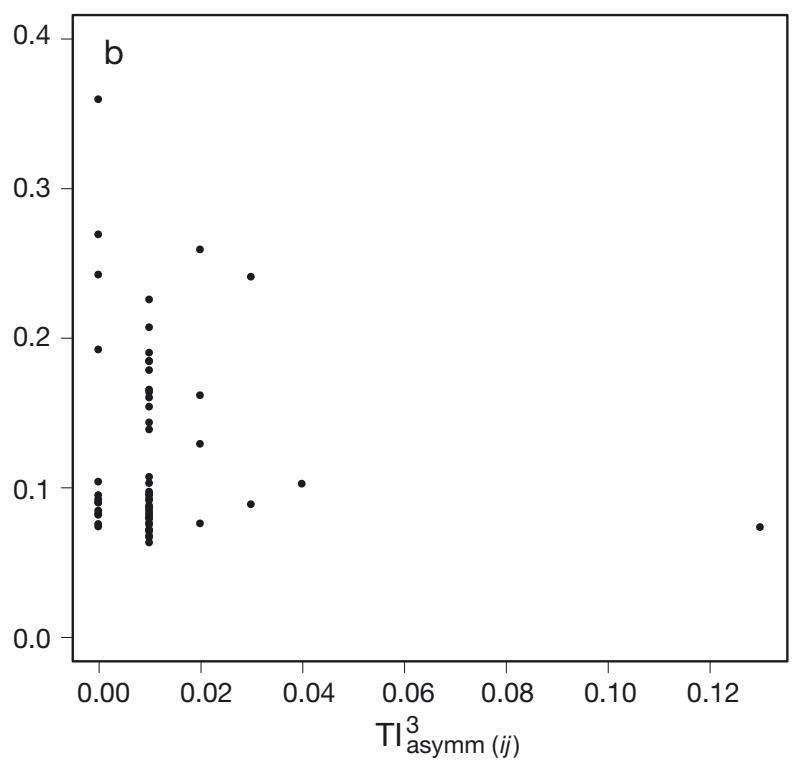

Fig. 4. Relationship between the simulated asymmetry $\left(C_{i j}\right)$ of $i j$ and $j i$ pairs of interactions and (a) the largest value of each pair of $e_{i j}$ and $e_{j i}$ effects (max $e_{i j i}$ Spearman's rho = 0.918 ; $\mathrm{p}<0.001)$, (b) structural asymmetry $\left(\mathrm{TI}_{\text {asymm(ij); }}^{3}\right.$ Spearman's rho $=-0.002 ; \mathrm{p}=0.986$ ), and (c) the stronger corresponding entries $\left(\max m_{i j}\right)$ in the mixed trophic impact matrix ([M]; Spearman's rho $=-0.103 ; \mathrm{p}=0.451$ ). See 'Methods' for definitions of terms

\section{DISCUSSION}

Even if we have more and more information about the strength of trophic interactions between prey and predators (e.g. McCann et al. 1998), it still remains unclear who is really influencing whom in a pairwise interaction. Beyond recognizing strong interactions, we also need to understand more precisely whether the strength is symmetrical in bottom-up and topdown directions. We performed food web simulation experiments and, following sensitivity analysis, determined each ij effect between pairs of species. We also quantified the asymmetry of $i j$ and $j i$ interactions and considered the most asymmetrical relationships (i.e. top $5 \%$ ). We then studied the graph of these dominant effects. As an illustrative case study, we 

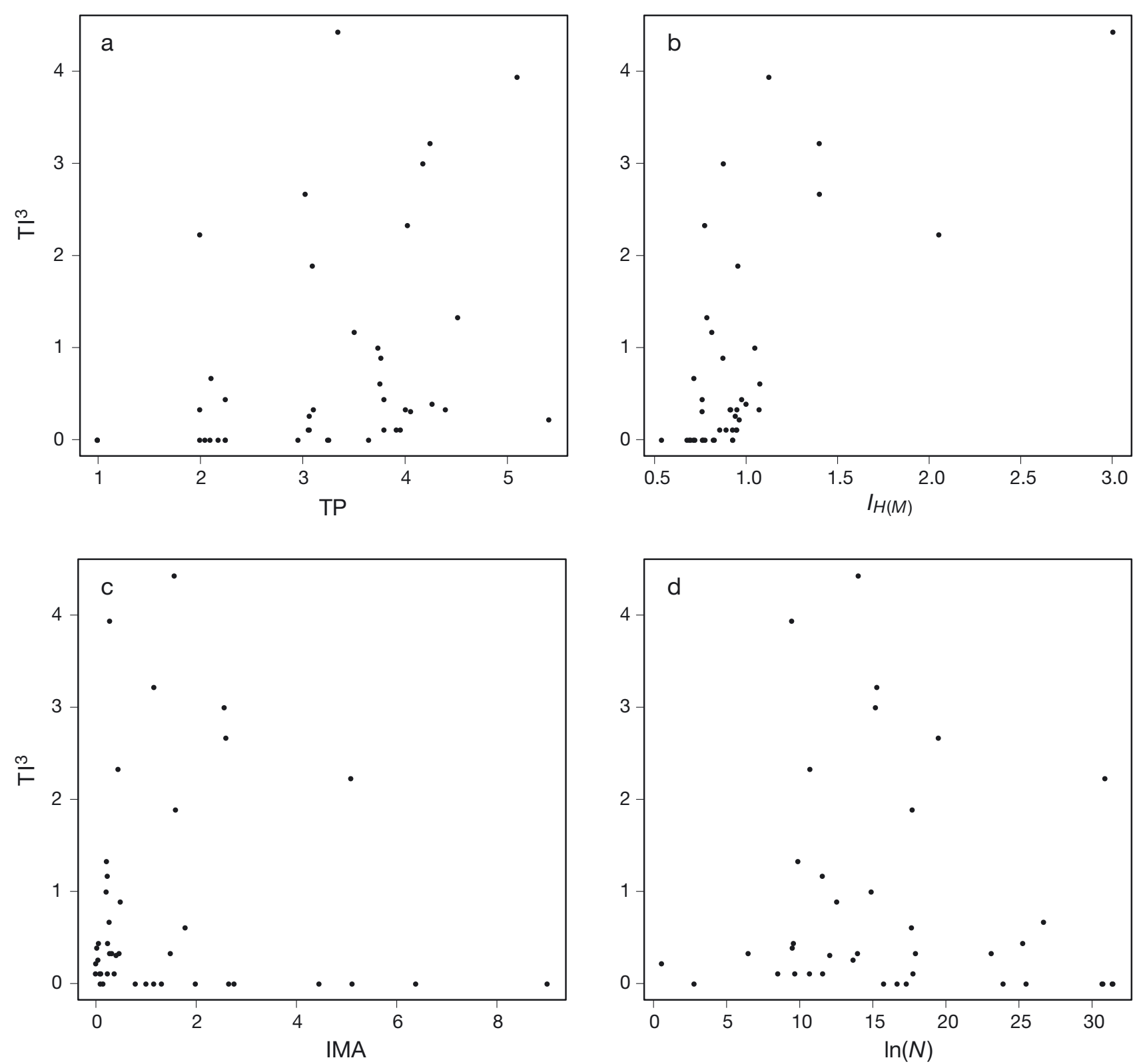

Fig. 5. Relationship between the directed topological importance up to 3 steps $\left(\mathrm{TI}^{3}\right)$ of species in the asymmetry graph and their (a) trophic position (TP; Spearman's rho $=0.494 ; \mathrm{p}=0.001)$, (b) relative importance based on the stochastic simulations $\left(I_{H(M)}\right.$ S Spearman's rho $\left.=0.588 ; \mathrm{p}<0.001\right)$, (c) cumulative effect estimated from mixed trophic impact matrix (IMA; Spearman's rho $=-0.116 ; p=0.466)$, and (d) the natural logarithm of their population sizes $(\ln (N) ;$ Spearman's rho $=-0.204 ; \mathrm{p}=0.214 ; N$ is the number of individuals)

used the weighted trophic flow network of the Prince William Sound ecosystem (Okey \& Wright 2004).

The suggested method is just one of several approaches that could be applied to identify and quantify the relative importance of inter-specific effects in ecosystems (as an alternative method, see mixed trophic impact, Ulanowicz \& Puccia 1990, also used for conservation purposes: Bondavalli \& Ulanowicz 1999). The main feature of our $C_{i j}$ index and the $\mathrm{TI}^{3}$ values of nodes in the asymmetry graph is that these clearly show the most understandable effects (i.e. strongly asymmetrical interactions) between species. Strong links identified by other tools (e.g. path analysis, Wootton 1994) may be symmetrical (both ij and $j i$ are strong), and in these cases it may remain unclear what exactly the mechanistic details of the relationship are.

The most central species in the asymmetry graph of the ecosystem (high directed $\mathrm{TI}^{3}$ ) feed at higher trophic levels, but are not necessarily top predators. 
This suggests a top-down control type of dominance, at least in the food web studied here. The species most influenced by others (low directed $\mathrm{TI}^{3}$ ) are found at lower than average trophic levels (Fig. 5a). This might imply a general top-down control in the ecosystem. Species seem to belong to 2 clusters based on the relationship between their importance and simulated relative importance (Fig. 5b): the few really important interactors are also relevant, while the mass of other species shows minor differentiations and less significance.

The finding that large populations may (e.g. adult salmon, adult arrowtooth) or may not (e.g. capelin) be very important in the asymmetry graph is interesting (see Fig. 5d), especially because we also recognize small populations of surprisingly high importance (e.g. transient orca, salmon shark). The generally positive relationship between population size and importance sounds quite intuitive from an ecological point of view, but it may seem counterintuitive for conservation practice: in conservation parlance, 'rarity' is often used as an equivalent of 'importance.' We still need to learn a lot about the relationship between rarity and importance, and we suggest that computational approaches may be helpful for a community-based characterization of the role of rare species.

Future developments include using more advanced network approaches for better understanding of the structural correlates of asymmetrical relationships. The weighted network offers alternative approaches, for example, using weighted degree or the weighted topological importance index (WI) (Scotti et al. 2007). Also, it will be an important development to add nontrophic interactions to this purely trophic model, as their relevance is being increasingly recognized (Whipple \& Patten 1993, Vasas \& Jordán 2006). Finally, here we did not consider the strength and importance of self-loops. This would be essential in the future, especially because there are important relationships between interaction symmetry, feedback, and self-loops.

This approach, focusing only on the graph of the most asymmetrical relationships, was proposed in the spirit of Lindeman (1941 see his 'basic dependencies'). It offers a simplified view on the most predictable, understandable effects dominating eco-dynamics and helps to single out the possibly clearest relationships in the community.

Acknowledgements. We are very grateful to the Editors for the invitation to submit this paper and to the anonymous reviewers for their excellent comments. Thomas A. Okey is kindly acknowledged for providing the database and earlier discussions. We thank Cinzia Ungari and Federico Vaggi for correcting the language and editing the manuscript. The research of F.J. was supported by the European Union and the State of Hungary, co-financed by the European Social Fund in the framework of TÁMOP 4.2.4.A/2-11-1-2012-0001 'National Excellence Program.'

\section{LITERATURE CITED}

Allesina S, Bodini A (2004) Who dominates whom in the ecosystem? Energy flow bottlenecks and cascading extinctions. J Theor Biol 230:351-358

Baird D, Ulanowicz RE (1993) Comparative study on the trophic structure, cycling and ecosystem properties of four tidal estuaries. Mar Ecol Prog Ser 99:221-237

- Bodini A (1991) What is the role of predation on stability of natural communities? A theoretical investigation. Biosystems 26:21-30

Bond WJ (1994) Keystone species. In: Schulze ED, Mooney HA (eds) Biodiversity and ecosystem function. Springer, Berlin, p 237-253

Bondavalli C, Ulanowicz RE (1999) Unexpected effects of predators upon their prey: the case of the American alligator. Ecosystems 2:49-63

Borrett SR (2013) Throughflow centrality is a global indicator of the functional importance of species in ecosystems. Ecol Indic 32:182-196

> Briand F, McCauley E (1978) Cybernetic mechanisms in lake plankton systems: how to control undesirable algae. Nature 273:228-230

> Brose U, Berlow EL, Martinez ND (2005) Scaling up keystone effects from simple to complex ecological networks. Ecol Lett 8:1317-1325

Christensen V, Walters CJ (2004) Ecopath with Ecosim: methods, capabilities and limitations. Ecol Model 172:109-139

> Dematté L, Priami C, Romanel A (2008) The Beta Workbench: a computational tool to study the dynamics of biological systems. Brief Bioinform 9:437-449

Estrada E (2007) Characterisation of topological keystone species: local, global and 'meso-scale' centralities in food webs. Ecol Complex 4:48-57

Gjata N, Scotti M, Jordán F (2012) The strength of simulated indirect interaction modules in a real food web. Ecol Complex 11:160-164

Hirata H, Ulanowicz RE (1985) Information theoretical analysis of the aggregation and hierarchical structure of ecological networks. J Theor Biol 116:321-341

> Hurlbert SH (1997) Functional importance vs keystoneness: reformulating some questions in theoretical biocenology. Aust J Ecol 22:369-382

Jones CG, Lawton JH (eds) (1995) Linking species and ecosystems. Chapman \& Hall, London

> Jordán F (2009) Keystone species in food webs. Philos Trans R Soc Lond B Biol Sci 364:1733-1741

Jordán F, Liu WC, van Veen FJF (2003) Quantifying the importance of species and their interactions in a hostparasitoid community. Community Ecol 4:79-88

> Jordán F, Okey TA, Bauer B, Libralato S (2008) Identifying important species: a comparison of structural and functional indices. Ecol Model 216:75-80

> Jordán F, Liu WC, Mike Á (2009) Trophic field overlap: a new approach to quantify keystone species. Ecol Model 220:2899-2907 
Jordán F, Scotti M, Priami C (2011) Process algebra-based models in systems ecology. Ecol Complex 8:357-363

Kazanci C, Matamba L, Tollner EW (2009) Cycling in ecosystems: an individual based approach. Ecol Model 220: 2908-2914

Lai SM, Liu WC, Jordán F (2012) On the centrality and uniqueness of species from the network perspective. Biol Lett 8:570-573

Libralato S, Christensen V, Pauly D (2006) A method for identifying keystone species in food web models. Ecol Model 195:153-171

Lindeman RL (1941) Seasonal food-cycle dynamics in a senescent lake. Am Midl Nat 26:636-673

Lindeman RL (1942) The trophic-dynamic aspect of ecology. Ecology 23:399-417

Livi CM, Jordán F, Lecca P, Okey TA (2011) Identifying key species in ecosystems with stochastic sensitivity analysis. Ecol Model 222:2542-2551

Luczkovich JJ, Borgatti SP, Johnson JC, Everett MG (2003) Defining and measuring trophic role similarity in food webs using regular equivalence. J Theor Biol 220:303-321

McCann K, Hastings A, Huxel GR (1998) Weak trophic interactions and the balance of nature. Nature 395:794-798

Melián CJ, Vilas C, Baldó F, González-Ortegón E, Drake P, Williams RJ (2011) Eco-evolutionary dynamics of individual-based food webs. Adv Ecol Res 45:225-268

Okey TA (2004) Shifted community states in 4 marine ecosystems: some potential mechanisms. PhD dissertation, University of British Columbia, Vancouver, BC

Okey TA, Pauly D (eds) (1999) A trophic mass-balance model of Alaska's Prince William Sound ecosystem, for the post-spill period 1994-1996, 2nd edn. Fisheries Centre Research Report 7(4), University of British Columbia, Vancouver, BC

Okey TA, Wright BA (2004) Toward ecosystem-based extraction policies for Prince William Sound, Alaska: integrating conflicting objectives and rebuilding pinnipeds. Bull Mar Sci 74:727-747

Ortiz M, Levins R (2011) Re-stocking practices and illegal fishing in northern Chile (SE Pacific coast): a study case. Oikos 120:1402-1412

Ortiz M, Wolff M (2002) Application of loop analysis to benthic systems in northern Chile for the elaboration of sustainable management strategies. Mar Ecol Prog Ser 242: 15-27

Ortiz M, Levins R, Campos L, Berrios F and others (2013) Identifying keystone trophic groups in benthic ecosystems: implications for fisheries management. Ecol Indic 25:133-140

Submitted: November 11, 2013; Accepted: July 11, 2014
Power ME, Tilman D, Estes JA, Menge BA and others (1996) Challenges in the quest for keystones. Bioscience 46: 609-620

Rooney N, McCann KS (2012) Integrating food web diversity, structure and stability. Trends Ecol Evol 27: $40-46$

Rooney N, McCann K, Gellner G, Moore JC (2006) Structural asymmetry and the stability of diverse food webs. Nature 442:265-269

Scotti M, Jordán F (2010) Relationships between centrality indices and trophic positions in food webs. Community Ecol 11:59-67

Scotti M, Allesina S, Bondavalli C, Bodini A, Abarca-Arenas LG (2006) Effective trophic positions in ecological acyclic networks. Ecol Model 198:495-505

Scotti M, Podani J, Jordán F (2007) Weighting, scale dependence and indirect effects in ecological networks: a comparative study. Ecol Complex 4:148-159

Scotti M, Gjata N, Livi CM, Jordán F (2012) Dynamical effects of weak trophic interactions in a stochastic food web simulation. Community Ecol 13:230-237

Seitz AC, Loher T, Nielsen JL (2007) Seasonal movements and environmental conditions experienced by Pacific halibut in the Bering Sea, examined by pop-up satellite tags. Scientific Report No. 84. International Pacific Halibut Commission, Seattle, WA

Ulanowicz RE, Kemp WM (1979) Toward canonical trophic aggregations. Am Nat 114:871-883

Ulanowicz RE, Puccia CJ (1990) Mixed trophic impacts in ecosystems. Coenoses 5:7-16

Vasas V, Jordán F (2006) Topological keystone species in ecological interaction networks: considering link quality and non-trophic effects. Ecol Model 196: 365-378

> Vasas V, Lancelot C, Rousseau V, Jordán F (2007) Eutrophication and overfishing in temperate nearshore pelagic food webs: a network perspective. Mar Ecol Prog Ser 336:1-14

Wasserman S, Faust K (1994) Social network analysis. Cambridge University Press, Cambridge

- Whipple SJ, Patten BC (1993) The problem of non-trophic processes in trophic ecology: toward a network unfolding solution. J Theor Biol 163:393-411

Wootton JT (1994) Predicting direct and indirect effects: an integrated approach using experiments and path analysis. Ecology 75:151-165

Yodzis P, Winemiller KO (1999) In search of operational trophospecies in a tropical aquatic food web. Oikos 87: $327-340$

Proofs received from author(s): August 17, 2014 